\title{
AI and the resurrection of Technological Determinism
}

This paper elaborates on the connection between the AI regulation fever and the generic concept of Social Control of Technology. According to this analysis, the amplitude of the regulatory efforts may reflect the lock-in potential of the technology in question. Technological lock-in refers to the ability of a limited set of actors to force subsequent generations onto a certain technological trajectory, hence evoking a new interpretation of Technological Determinism. The nature of digital machines amplifies their lock-in potential as the multiplication and reuse of such technology is typically almost cost-free. I sketch out how AI takes this to a new level because it can be software and an autonomous agent simultaneously.

Keywords: artificial intelligence; technological determinism; social control of technology; AI ethics.

\section{Acknowledgements}

The research was supported by the János Bolyai Research Scholarship of the Hungarian Academy of Sciences

\section{Author Information}

Mihály Héder, Budapest University of Technology and Economics; SZTAKI Institute for Computer Science and Control https://orcid.org/0000-0002-9979-9101

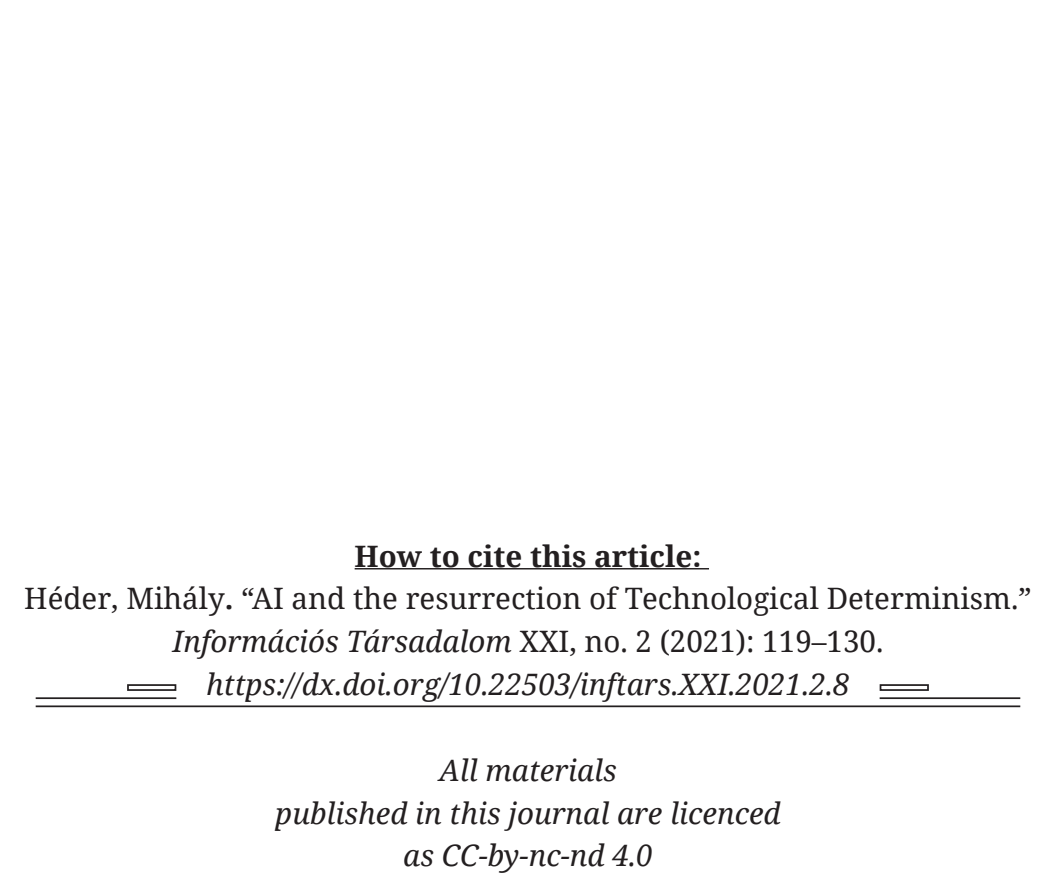




\section{Introduction}

In this paper I argue that the current wave of Artificial Intelligence Ethics Guidelines can be understood as desperate attempts to achieve social control over a technology that appears to be as autonomous as no other. While efforts at the social control of technology are nothing new, AI with its unique nature may very well be the most resistant to such control, which validates the amount of attention the question receives.

However, should regulatory attempts fail, future society may be determined by the nature of this technology, dread many thinkers. There is an attitude/historiographic methodology called "technological determinism", which has been widely criticized and almost completely dissected since the second half of the 20th century. This attitude is recurrent again in the case of AI, and perhaps has found a more solid footing there.

One pillar of technological determinism is a perceived inevitability about the direction of technological progress, which, like gravity, tends towards ever higher efficiency, and trying to resist it for long is a fool's gambit. The other pillar is that this predetermined nature of technological evolution acts as an exogenous force on society and causes it to change. In other words, technology progresses following its own internal logic and society is restructured as a side effect of this. Consequently, humanity trades its potential for being a Being for an Iron Cage, where only mass-produced Whipped Cream is available but not the real thing. ${ }^{1}$

Social scientists and critically minded philosophers ever since the sociological turn - sometime around the sixties - came up with one case study after another that all showed the surprising causal powers of persons or groups of people on the trajectory of technology. These investigations indicated the reverse of the deterministic view. It appeared that the idiosyncratic decisions of some humans - rooted in their culture, world view, office politics and other factors of this kind, but not in technological reasoning - acted as an exogenous force on technology, rather than the other way around.

For the sake of understanding the relationship between AI and society, this article reconstructs the technological determinist position and investigates the aftermath of the technological determinism debate. On the surface, it might appear that the case is closed and social constructionism has won; at least that the stronger formulations of technological determinism cannot be maintained against the decisive evidence from several case studies. Yet, the general attitude of the deterministic view seems to be resurfacing in discussions around climate change, the effects of social media, and so on. Maybe this is only because of the lack of awareness of the determinism debate and its outcome. But could it also be that some of these technological trajectories - AI being one - are different?

After reviewing the technological determinism landscape, we venture further to examine the notions of technological lock-in, the irreversibility of technology and the existential risk of technology. These seem to suggest that while technology may be indeed socially constructed at a given point in time, later generations have limited freedom in re-interpreting it or phasing it out. In this way, technology may be-

\footnotetext{
${ }^{1}$ The author apologizes for the conflation of references to Martin Heidegger (1952), Max Weber (1904) and Albert Borgmann (2003).
} 
come an inter-generational tool of power by which earlier generations determine some important aspects of - and even limit the boundaries for - later societies: a possible new type of technological determinism.

\section{The fundamentals of Technological Determinism}

Technological determinism refers to the notion that technology shapes society and culture. There is no canon definition for this, rather there are several versions that share a family resemblance with each other. Arguably, the most extreme, hard form of technological determinism, in which there is no place for social control, is quite difficult to defend and as a result it would be hard to find even a handful of serious proponents for it. But the non-existence of the phenomenon is equally implausible. Therefore, technological determinism concepts must be distributed on a scale between these two extremes of full determinism and full indeterminism.

As we look for common features among the several formulations of technological determinism, we will find a claim about causation and another about imbalance. The first claim considers how a given technology - or sometimes the technologically modern state of affairs in general, i.e. a technological milieu (Ellul 1964) - can be a cause and a feature of an aspect of society (usually a negative one, like the loss of freedom) that arises as an effect of that cause.

The notion of determinism should evoke certain metaphysical concepts. Indeed, some metaphysical theories argue for a completely deterministic view of the universe and therefore every feature of it. Most variants of these theories, and certainly the technological one, are causal determinisms.

If we attempted to interpret technological determinism in this wider framework, perhaps we could point to some causal chains of such a world, in which the role of a technological artefact precedes a change in a feature of society, and hence the cause-and-consequence sequence would be established. The problem of the deterministic world is often discussed in the debate around free will.

But this is usually not how a technological deterministic debate is structured. Instead, such debate tends to sidestep the metaphysical question, or at least assume a world where freedom is at least a theoretical possibility, not dive into the question of whether this means an indeterminate world or a compatibilist (where determinism and freedom can coexist) universe.

While not engaging with the free will problem, the critiques of technological determinism tend to rely on another concept of modern philosophy, namely underdetermination, and the Duhem-Quine thesis of it (Bloor 1991). However, for the purposes of this chapter, it is important to note that underdetermination is primarily considered an epistemic concept.

Besides the freedom of people, as a value that is worth being worried about, debate around technological determinism also often includes a notion of the autonomy of technology. This notion of autonomy is even less contrasted with the fundamental question about the deterministic or indeterministic nature of the world as much as the freedom of people. Instead, the autonomy of technology is discussed at the level 
of history and of society, as a relative term, as in technology is free from human control, even in Latour's actor-network theory (Latour 2013), where he introduces elements of science and technology as non-human agents.

The second claim, the one about imbalance, grants stronger, more dominant causal powers for technology in comparison with society or culture. This clause is necessary, because it is evident that consumer behaviour, the inventor's and corporate decisions, technology regulation and other venues of human agency - and therefore social control - do have a causal effect on technological artefacts and technological development. Since that is hard to deny, a technological determinist position needs to claim that the role of technology is still more dominant. In spite of all the factors above, this tends to be the decisive factor. This is why, in a technological determinist view, the nature or essence of technology has such big importance: that its essence will eventually manifest itself in the character of society.

However, the case studies from STS and other historic accounts serve as convincing arguments that there is not much point in talking about this issue in very broad, generic terms. Except from some extreme forms of the technological determinist position, the determining powers of technology should differ from case to case, place to place and perhaps even between different historical ages. So a well-formulated technologically deterministic position should state which particular technology has a causal effect on which particular feature of society or culture, instead of making categorical claims about the supremacy of technology in general.

This does not mean that general claims cannot be found. Ellul's (1964) technological milieu concept discusses technologically advanced societies, while occasionally showing some concrete examples of the stated problems. Feenberg (2009) also operates with a concept of technological hegemony, which serves as an ambient background that is beneficial for the causal powers of technology. In this respect his position is similar to Borgmann (2003), who also discusses general tendencies, albeit in a very nuanced manner.

Also, the versions of the theory vary around the role of different groups of people. It is possible to construct theories in which technological, political or economical elites escape being determined by technology, or they may even determine the life of others through technology. Based on this differentiation, the elements of a technologically deterministic theory are often found in political philosophies, like Marxism and its successors, that partially reject and partially elaborate it, like the Frankfurt school, just as well as in other technocratic views of the world.

The stakes of this question are very high since the answer obviously is an input for social organization. A view of the possibilities of taming technology can reinforce our approach to AI among other high-potential technologies.

\section{Cases of social control}

There are several supposed examples of technological determinism throughout history. One that is widely stated is the effect of the invention of the printing press on the politics of organized religion on the European continent, or simply put, how 
book printing led to reformation. Another example has to do with the invention of the stirrup and feudalism. Yet another concerns gunpowder technology and the colonization of the world by European empires. These accounts of course do not withstand the scrutiny of a more detailed economic-sociological analysis. They usually neglect the possibility of the sociological context having an equally large causal effect on the invention than the invention has on the society. As pointed out in the previous section, in technological determinism it is implied that technology is the dominant force, not the co-evolution of science and technology as equals.

Another issue with these historic examples is that they do not report on negative cases equally, hence violating the well-known principle of symmetry. Bijker, Hughes and Pinch (1992) and other social constructionists of technology use this methodological maxim, inherited and adapted from the strong programme, to describe the necessity of treating technological failures and successes with equal attention. In our context, this would mean contrasting those cases in which a technological breakthrough apparently led to social change with those other cases where a similar technological advancement did not have the same effect.

The use of gunpowder is a very good example for highlighting the need to consider social factors. Pioneered in medieval China, and later adapted by Japan, the Ottoman Empire, the Russian Empire and many others, it did not lead to the same social transformation in those regions as in Europe (Hoffman 2012). So other factors must have been at play. This evidences the necessity for the presence of certain social conditions for change to happen.

If that is the case, we cannot think about these issues with a monocausalistic model anymore. That is, we cannot further maintain that technology is the sole cause of change in these cases, and if it has to share this role with several social factors; thereby the dominance of technology, as encapsulated in the technological determinism concept, is again lost.

And in truth, the social factors are plentiful. Religion and ideology is one obvious candidate to enhance or hinder the acceptance of technological change. Wage levels are often seen as a necessary condition for labour-saving capital expense. War is often cited as a catalyst of technological breakthroughs; albeit if all for the wrong reasons. Also technological determinism not only has to share its influence with social factors but possibly with other forms of determinisms too. For instance, geographical determinism suggests that being on a certain spot on the planet may be decisive. The inhabitants of Easter Island had to face challenges because of the nature of their habitat, just as the Europeans who were denied access to Middle-Eastern trading routes, or the British with access to coal but with the necessity to pump out the water from the shafts; for which, steam power proved to be handy.

Most thinkers when confronted with the implausibility of the extreme positions around technological determinism tend to seek a middle ground. Some thinkers consider their position more in line a form of soft technological determinism (Dusek 2006, Heilbroner 1967).

Another way to find the middle ground is through considering the concept of underdetermination, as Andrew Feenberg does. This solution is especially interesting as it focuses on the co-causal powers of technology and human agency. This view 
allows for a theoretician to appreciate the difference between a passive and an techno-politically conscious society.

Feenberg acknowledges that technology, if left alone, has inherently anti-democratic tendencies. He further claims that as more and more social activities become mediated by technology, those tendencies will gain more room to flourish. Therefore, if technology is left alone - instead of actively developing a critical view about it - our freedom will indeed diminish. This is why Feenberg argues for actively injecting democracy into technology and into the technologically mediated areas of life (which are more and more as time progresses); even in areas that were previously thought off-limits for democratic decision-making, like in a factory.

However, Feenberg argues, this really needs to be actively pursued, in order to avoid a natural tendency of society towards becoming ever-more technocratic, and hence less democratic. This means that in his model of the world, change will still happen, but without an active, conscious agency of humans, but also, that without the timely, active participation, our window of opportunity may be lost for ensuring control of that change. Based on this framing, Stump (2006) categorizes Feenberg's view as one that still involves the essentialism of technology.

While Feenberg never uses the following particular terminology from the philosophy of technology, the possibility he explores depends on the co-causation model of social change. In this, there is room for humans to work as a causal component to counterbalance the anti-democratic causal component that technology represents.

\section{The Social Construction of Technology}

By describing society as a co-causative factor, we can overcome another deterministic concept, the supposed "trade-off" situation of technology adoption. This considers that society has to make a tough decision about technology: it either uses the technology and suffers its side effects, or it does not adopt it and may be harmed by missing out on the potential advantages and economic growth the technology could bring. This description of the technology adoption problem makes society look external to the technological change; in effect, a mere bystander that needs to make up its mind about a new situation it may find itself in.

The contrary of the trade-off view is Constructivism, or the Social Construction of Technology (SCOT). This position sees the direction of technological change as being underdetermined by mathematics, the laws of nature, or other non-negotiable features of our universe. And if this is undetermined, it means there is room for society to manoeuvre. It has to be noted, that instead of the concepts of underdetermination and co-causation, as commonly used in the philosophy of science, the sociologists who explore this situation tend to rely on expressions from the philosophy of language. As a result, technology is subject to "interpretation" in this terminology. The outcome of this interpretation, of course, is to a great extent up to the users of the language. Translating the analogy for the question at hand yields that the outcome of technological change is up to the makers and users of the technology. 
Yet another linguistic concept is the hermeneutics of technology: an iterative interpretation process that society exercises when adopting a new technology. In the context of this process, technical objects have two hermeneutic dimensions. Their social meaning, which is established in a manner that may even be called argumentative, and this defines what kind of role an object may play in the lifestyles of its users. This approach counters the second dimension related functionalism, which considers objects in an inherently de-contextualized manner and views technical objects as neutral means to achieving certain ends that are fundamentally external to the object. Feenberg, by re-contextualizing technology in its social environment, breaks down a hidden assumption behind technological determinism: that rationality is culture-independent.

And if that is not the case, it is also impossible that the trajectory of technological development, which supposedly is about always picking the most rational means to achieve ever-increasing efficiency, is set in the stars. Such rationality will now depend on the social context, and at this point, democratic rationalization is a straightforward possibility and just requires a cultural preference for technological development.

The important conclusion of the above arguments for the possibility of social control over technology is that when it comes to the ontology of technical artefacts, we cannot maintain that one kind of aspect - like functionality or rationality in reaching a goal - is inherent, while other kinds - like social meaning or preferences - are assigned just in the observer's mind. Instead, we must conclude that these aspects are equally essential to the given artefact. This is the "double aspect theory". If there is an unreflexive interpretation process - that is, an interpretation that does not acknowledge that it analyzes the objects with completely idiosyncratic preconceptions of effectiveness - technology will indeed appear as an external force on society.

This raises the question on whether anything has actually fundamentally changed our "modern world", meaning our digital, virtual, and industrialized worlds. This question is crucial, since humanity has always been technical - in fact, elaborated tool usage is a common milestone in the historical accounts of the evolution of our species.

\section{Increasingly powerful technologies}

In the first waves of the technological determinism debate, the autonomy of technology meant an abstract situation in which the nature of technology keeps relentlessly manifesting itself though the rationalization efforts of humans. We saw how the double aspect theory questioned whether this is inevitable. However, what if a technology - several instances of it to be more precise - is more literally autonomous, like AI? My argument is that in this case we have to deal with technology of a different nature, rendering most of the constructionist arguments irrelevant. But before we get there, it is best to build up a picture through considering other, equally recent, technological achievements.

Take social media. In the 2020s, it is a common argument that the nature of political campaigning has drastically changed thanks to this technology and that its users 
grip on reality may already be incorrigibly broken. This is a picture in which the medium dominates the discourse, which has fuelled renewed interest in the works of McLuhan, somebody who is usually categorized as a technological determinist, but who later in his career softened his stance somewhat.

On the internet, and particularly social media, there is support for seemingly any claim no matter how far-fetched with reports on evidence that would seemingly corroborate it. This is just another form of increase in control - paradoxically, we seem to be able to control and entrench our own beliefs even by tendentiously selecting the content we consume. But the control is not evenly distributed, it is affected by AI and somewhat determined by our biology. In criticisms of social media, references about how we are being manipulated through targeting our dopamine centres are common.

The time dimension of being dominated this way is especially interesting. In the case of social media, it has been stated that it takes sustained conditioning over some amount of time to arrive at a drastically polarized society, in which the camps are not capable of having discourse anymore due to their incompatible perceived realities and semantics. At this point, the positions become so entrenched that it seems there is no way back anymore.

The time horizon appears to be even more important as we arrive at technologies that are able to change the environment, cause climate warming and environmental pollution in general. Here the urgency for action is derived from the predictions that the window of opportunity is closing - in fact, for the most positive scenarios it has already slammed shut. The immediate importance of this topic was already evident and the situation seemed already dire in 1999 when Feenberg's Questioning Technology (2009) dedicated a chapter to environmentalism and the surrounding politics. Andrew Light (2006) added interesting further thoughts to the debate that the chapter analyzes.

I think that the very urgency that everybody exhibits around the issue of the environment and global warming illustrates a realization of the possibility of irreversible negative change. But that irreversibility in turn means that the world is changing in a way that the arguments against technological determinism and on behalf of social control become less and less convincing.

This evokes a concept that is reasonably present in the management and history of technology but curiously underrepresented in science and technology studies.

The concept in question is technological lock-in.

Based on the terminology of the previous sections, we can summarize technological lock-in as a process where the possibility of change technology is gradually lost as the window for modifications becomes closed.

There are multiple reasons for this. David (1985) identifies technological co-dependence, economies of scale and irredeemable investments as key reasons. This is expanded by Cowan and Hultén (1996) with several new factors, like the necessity of a crisis, regulation, and technological breakthroughs for changing an incumbent technology, while the lack of these means the status quo being sustained. Foxon (2014) further elaborates the role of institutions and the epistemic aspect in general.

It seems logical that a concept of irreversibility is necessary for explaining how 
the unfortunate situation of technological lock-in may occur. In the next section, we take the case of irreversibility to the extreme.

\section{AI enters the scene}

As the presence of new technology in our everyday life increases, sometimes the general public may suddenly become alarmed by its towering presence. It is not clear when exactly this happens. For instance, in the last years of the 2010s several regulation efforts all around the world were launched to handle the ethics of Artificial Intelligence. Global institutions like UNESCO, professional bodies like the IEEE (2019) and the European Union (2020), and several other organizations and companies made declarations in this area in or close to 2019 (Héder 2020). Their urgency appeared quite similar to what we are experiencing around global warming, and I argue that the reason is the same: AI has a tremendous lock-in potential. ${ }^{2}$

There are several factors that make AI especially prone to being locked in.

First, AI is software. Like with any software, the cost of "manufacturing" - producing more instances of the same design, which is copying in this case - is ridiculously low; indeed in most cases, completely negligible. And yet, a profit may be realized on each "unit" or licence, meaning that creating well-received software can be extremely lucrative - write once, derive profits over and over again. Indeed, the most successful track for social mobility seems to be creating and owning software and related IT. Many of the wealthiest people in the world, unless inheritance was in play, rose up by developing some successful software - think of Gates, Bezos, Musk, Zuckerberg, etc. And, of course, thanks to the internet, not only "manufacturing" but also "delivery" (downloading) is basically costless with software.

This means that if in the future a problem class is quite successfully solved by a piece of AI software that is also reasonably available - free or cheap, considering the value - then there will not be much incentive to develop alternatives. In reality, this rarely means a complete monopoly over a problem class and there is always a small number of commercial and some open-source competitors, but if we think about it, many categories of software today are covered by extremely few options. Think about the number of pdf readers or web browsers you use. There is more than one, but the list ends surprisingly quickly, and there is also the fact that some of these are really the same under the hood, but with different interfaces.

If the software in question is also free and open source with a licence that is compatible with most interests, the dominance of one single solution can become extreme. A case in point is the Linux kernel that is present in any android-powered device and that serves the overwhelming majority of web pages and can be found in billions of smart appliances. It really is like a stick-and-carrot situation - writing your own operating system is insurmountable except for the largest institutions, while on the other hand, reusing what is already there is free. Now, this only means

\footnotetext{
${ }^{2}$ There are, of course, other theories as to explain the sudden surge in AI ethics, like the extension of Politics to regulation (Gyulai and Ujlaki 2021), or simple „ethics washing” (Vică et al. 2021).
} 
that the people that have a say in the development of the software in question have an oversized control over an entire industry, so they need to be engaged on various platforms in order to achieve the social control of technology. However, the situation is worse than that. In fact, many of these projects are inter-generational and the current shepherds of any single technology may have limited control over the trajectory of software, especially if the software is already ubiquitous and any significant rewrite would require more effort than the current generation can offer.

On top of the digital nature of software - which I argue, is enhancing its lockin propensity - there is now the phenomenon of Software-as-a-Service (SaaS, or, vaguely, the "cloud"), which mobilizes economies of scale, in this case for data. This elevates the lock-in potential of software to an entirely new level. By aggregating several users and use cases, companies offering SaaS can leverage the network effect between those users for their own benefit. While copying and delivering software is negligibly cheap, there is still a cost to using non-SaaS software, mainly installation and maintenance costs. With SaaS, these costs, too, are greatly reduced. This creates situations akin to natural monopolies: the author of this paper surveys his Ethics of AI students each semester, and always finds that there is a $100 \%$ penetration of Gmail among the students surveyed. This is despite the fact that really absolutely nothing prevents anyone from running a similar service.

The already unusually ample lock-in potential of the combination of software with the internet (SaaS) is further enhanced by a particular feature of AI: the need for data for machine learning. Artificial Intelligence delivered as SaaS has a unique potential that no other distribution method can match. Therefore, we can expect, with some confidence, that whenever a SaaS AI becomes sufficiently good in the targeted problem space - e.g. a translator or proofreader solution - then it will become simply uneconomical to compete against it.

Finally, this picture would be completed with the possibility of a self-enhancing, ever-more autonomous SaaS AI, which is really one of the promises of machine learning. This would enable over time opening a gap between any new contenders and an established solution in a problem space - for the benefit of the incumbent.

An autonomous - which in this case only means self-driven, proactive intelligent behaviour - AI agent present entirely different problems for social control. Regardless of what phenomenological state we ascribe to such an agent, the interactive nature of such machines will make them actors rather than mere objects. Suddenly, in the debate around technological determinism, these agents may appear on the other side of the equation, the one that has so far been reserved for humans only. And this truly counts as the resurrection of the technological determinism debate.

\section{Discussion}

This article summarized some of the positions around technological determinism for understanding the reception of contemporary AI. To analyze the various shades of technological indeterminism and social control, we used - sometimes inspired by the STS literature itself - the terminology from the philosophy of science, namely the 
epistemic concept of underdetermination, the criticism of monocausation and the arguments for co-causation in place of it.

However, the Quineian arguments for the undefeatable underdetermination of theories by empiria are not arguments for the underdetermination of change by all the factors that we cannot control. In fact, there is no guarantee that all the relevant processes we care about - for instance, change in society - will always be controllable as well.

In this article, I explored a dynamic view of the balance between the primacy of technological and social factors. Specifically, I posited the question of whether this may shift over time, and not to the advantage of society. This idea of course is nothing new: irreversible environmental change and technological lock-in have both been commonly discussed for several decades. It is a fair and existential question then whether the means of technological power and social control are in such an imbalance.

The nature of scientific knowledge and engineering knowledge - that it is easier to reuse than to discover, easier to copy than to design - suggests that it is easier to increase the general level of technological prowess than to decrease it. In other words, the margin cost of reusing knowledge is diminishingly low. Extrapolating this thought to digital technology, we found that AI is especially interesting, since the multiplication and reuse of such technology is typically almost cost-free.

I sketched out how AI could be such a technology, by the virtue of it being software, but more specifically Software-as-a-Service. This enhances the lock-in potential of AI as all the necessary conditions of technological lock-in are present: fast dissemination and an uncommonly strong economical factor for reuse instead of re-creation, turbo-charged with the economies of centralized data collection for the sake of machine learning.

Finally, I touched on the question of whether technology can have actual agency, instead of the metaphorical agency the proponents of technological determinism have suggested before. This would mean AI agents appearing as relevant social groups in the shaping of their own trajectory, and thereby completely re-framing the debate of technological determinism.

\section{References}

Bijker, Wiebe, Thomas P. Hughes, and Trevor Pinch. "The Social Construction of Technological Systems.” In Shaping Technology/Building Society: Studies in Sociotechnical Change, edited by Wiebe Bijker and John Law, Cambridge: MIT Press, 1992.

Bloor, David. Knowledge and Social Imagery. Chicago: University of Chicago Press, 1991. Borgmann, Albert. Power failure: Christianity in the culture of technology. Baker Books, 2003. Cowan, Robin, and Staffan Hultén. "Escaping lock-in: the case of the electric vehicle." Technological forecasting and social change 53, no. 1 (1996): 61-79. 
David, Paul A. "Clio and the Economics of QWERTY." The American economic review 75, no. 2 (1985): 332-337.

Dusek, Val. Philosophy of technology: An introduction. Blackwell, 2006.

Ellul, Jacques. The Technological Society, trans. J. Wilkinson, New York: Vintage, 1964.

European Commission. On Artificial Intelligence - A European approach to excellence and trust. COM 65. Accessed: June 30, 2020. https://ec.europa.eu/info/sites/info/files/commissionwhite-paper-artificial-intelligence-feb2020 en.pdf

Feenberg, Andrew. Questioning Technology. Routledge, 1999.

Feenberg, Andrew. "Democratic rationalization: Technology, power, and freedom.” In Readings in the philosophy of technology, edited by David M. Kaplan, 139-155., Lanham, MD: Rowman and Littlefield, 2010.

Foxon, Timothy J. "Technological lock-in and the role of innovation." In: Handbook of sustainable development, edited by Giles Atkinson, Simon Dietz, Eric Neumayer, and Matthew Agarwala, Edward Elgar Publishing, 2014.

Gyulai, Attila and Anna Ujlaki. “The political AI: a realist account of AI regulation.” Információs Társadalom 21, no. 2 (2021). https://doi.org/10.22503/inftars.XXI.2021.2.3.

Heidegger, Martin. “The Question Concerning Technology.” (QCT), in The Question Concerning Technology and Other Essays, New York: Harper Collins, 1952.

Heilbroner, Robert. L. “Do machines make history?” Technology and Culture 8, no. 38 (1967): 335-45 (also in Scharff and Dusek, pp. 398-404).

Héder, Mihály. "A Criticism of AI Ethics Guidelines.” Információs Társadalom 20, no. 4 (December 31, 2020): 57-73. https://doi.org/10.22503/inftars.XX.2020.4.5.

Héder, Mihály. "The Epistemic Opacity of Autonomous Systems and the Ethical Consequences.” AI \& SOCIETY, (July 30, 2020b). https://doi.org/10.1007/s00146-020-01024-9.

Hoffman, Phillip T. "Why was it Europeans who conquered the world?” The Journal of Economic History 72, no. 3 (2012): 601-633.

IEEE Global Initiative on Ethics of Autonomous and Intelligent Systems. Ethically Aligned Design: A Vision for Prioritizing Human Well-being with Autonomous and Intelligent Systems, First Edition. Last Accessed: Dec 20, 2019. https://standards.ieee.org/content/ieeestandards/en/industry-connections/ec/autonomous-systems.html

Latour, Bruno. "Reassembling the social. An introduction to actor-network-theory.” Journal of Economic Sociology 14, no. 2 (2013): 73-87.

Light, Andrew. "Democratic technology, population, and environmental change." In Democratizing technology: Andrew Feenberg's critical theory of technology, edited by Tyler J. Veak, SUNY press, 2006.

Stump, David. "Rethinking modernity as the construction of technological systems." In Democratizing technology: Andrew Feenberg's critical theory of technology, edited by Tyler J. Veak, SUNY press, 2006.

Constantin Vică, Cristina Voinea, and Radu Uszkai. “The emperor is naked: moral diplomacies and the ethics of AI.” Információs Társadalom 21, no. 2 (2021): 83https://doi.org/10.22503/inftars.XXI.2021.2.6

Weber, Max. The Protestant Ethic and the Spirit of Capitalism, T. Parsons (trans.), A. Giddens (intro), London: Routledge, [1904] 1992. 